\title{
EXPANSION OF OPTICAL PROPERTIES IN TIOx NANOPARTICLES BY THEIR LASER- MEDIATED ALLOYING WITH AG
}

\author{
${ }^{1}$ David ETTEL, ${ }^{1}$ Ondrej HAVELKA, ${ }^{2}$ Selingul ISIK, ${ }^{1}$ Fatma YALCINKAYA, \\ ${ }^{1}$ Rafael TORRES-MENDIETA \\ ${ }^{1}$ Institute for Nanomaterials, Advanced Technologies and Innovation, Technical University of Liberec, \\ Liberec, Czech Republic, EU \\ ${ }^{2}$ Faculty of Textile, Technical University of Liberec, Liberec, Czech Republic, EU, Rafael.Torres@tul.cz
}

https://doi.org/10.37904/nanocon.2020.3700

\begin{abstract}
TiOx nanoparticles are large bandgap materials that attract a great deal of attention for their use in lightpowered applications. However, their utility is often restricted to employing energy from the ultraviolet (U.V.) or the second near-infrared solar spectrum region. In the current contribution, we expand the optical response of this material by its laser-mediated alloying with $\mathrm{Ag}$ nanoparticles, of which surface plasmon resonance activity allows the absorption of the visible solar spectrum region. For this, we produce the laser ablation of a $\mathrm{Ti}$ foil immersed in an aqueous solution of silver nitrate $\left(\mathrm{AgNO}_{3}\right)$. The analysis of the material using transmission electron microscopy, inductive coupled plasma mass spectroscopy, and UV-vis spectroscopy enabled recognizing that the use of the minimal $\mathrm{AgNO}_{3}$ solution of $1 \mathrm{mM}$ leads to the optimal synthesis of nanoalloys with the coexisting crystalline phases TiO (JPDS database: PDF 00-043-1296), and Ag (PDF 00004-0783), the bimodal size distribution centered at 60 and $130 \mathrm{~nm}$, the elemental composition of $31.22 \% \mathrm{Ag}$ and $68.78 \% \mathrm{Ti}$, and finally, the absorbance edges centered at 400 , and $638 \mathrm{~nm}$.

Unlike conventional methodologies, in the presented approach, no special conditions or hazardous reducing agents are required. Therefore, we believe the current work will be of great interest in the facile and wastereducing synthesis of nanoalloys composed by elements with contrasting optical properties.
\end{abstract}

Keywords: Nanoparticles, nanoalloys, Ti, Ag, Laser synthesis of nanoparticles

\section{INTRODUCTION}

In past years, nanomaterials research allowed the production of materials with unconventional optical properties compared to bulk materials [1]. One group of particular interest are nanoparticles (NPs) of Ti oxides, whose ability to generate photo-induced charge carriers upon exposure to light has led to their use in plenty of applications like photocatalysis, solar cell development, water purification, and photothermal conversion, among others [2]. Nevertheless, their real employability is often restricted to the percentage of the total electromagnetic spectrum that can absorb, e.g., in the case of $\mathrm{TiO}_{2}$, which is the most popular form of titanium oxide, its absorption is limited to the ultraviolet region [3], in the case of other titanium oxide forms like black $\mathrm{TiO}_{2}$ or $\mathrm{Ti}_{2} \mathrm{O}_{3}$, the absorption range gets extended to the visible and infrared regions [4]. However, their absorption efficiency is limited. The latest and most efficient trend to overcome these limitations is expanding their absorption capabilities through alloying with noble metal NPs [5]. These NPs display a phenomenon known as surface plasmon resonance (SPR), in which their free electrons in the conduction band oscillate collectively upon their exposure to light, and depending on the NPs size; electromagnetic waves with a specific wavelength resonates with the electronic oscillation. This phenomenon leads to its preferential absorption over the rest of the electromagnetic spectrum. When elements that experience SPR activity are mixed with materials 
like titanium oxides, synergistic effects can occur where photogenerated charge carriers can transfer from one element to the other, enhancing in this way the titanium oxide's harvesting of light [6].

The most straight forward ways of mixing these elements encompass either ball milling-based strategies or co-precipitation-based once, which often imply the use of special conditions, hazardous reducing agents, and large production of polluting by-products [7]. In the current contribution, we address these issues by the laser ablation of a $\mathrm{Ti}$ foil immersed in an aqueous solution of silver nitrate $\left(\mathrm{AgNO}_{3}\right)$. According to the relevant literature, when ablating a solid target in an aqueous medium, the detached material, including electrons, ions, and other energetic species, creates a plasma where all the forming species recombine, generating NPs [8]. In a traditional experimental procedure, the plasma's energy is released to the surrounding liquid medium forming a cavitation bubble where the NPs experience coalescence, reaching in this way their final size, and after the cavitation bubble extinguishes, the NPs get finally released to the liquid medium. However, when the liquid medium is filled with molecules that are precursors of other NPs, the plasma can render the precursor reduction/oxidation via charge transfer or through their interaction with sensitizers created by the solvent's molecular dissociation that surrounds the plasma (radicals or excited molecules) [9]. After the reduction/oxidation process occurs, the produced ions transfer into the plasma [10], resulting in nanoalloys' formation.

Since the proposed methodology enables the improvement of the optical capabilities in Ti oxide NPs by their mixing with $\mathrm{Ag}$, while at the same time does not require any hazardous chemical agents, special conditions and greatly suppresses the generation of by-products, it is believed that the current investigation will be of great value for the eco-friendly design of new materials with tailored optical properties

\section{EXPERIMENTAL}

The nanoalloy synthesis took place by the laser ablation of a Ti foil $(0.25 \mathrm{~mm}$ thickness, $99.7 \%$ trace metals basis, Sigma-Aldrich) immersed in $0 \mathrm{mM}, 0.001 \mathrm{mM}, 0.01 \mathrm{mM}, 0.1 \mathrm{mM}, 1 \mathrm{mM}$, and $10 \mathrm{mM}$ solutions of $\mathrm{AgNO}_{3}$, ( $\geq 99.0 \%$ pureness, Sigma-Aldrich) in distilled water $(18.2 \mathrm{M} \Omega \cdot \mathrm{cm})$. The Ti plate was irradiated by a High-energy industrial femtosecond laser (Onefive Origamy XP, NKT Photonics), which has a 400 fs pulse duration, a central wavelength centered at $1030 \mathrm{~nm}$, a repetition rate of $1 \mathrm{MHz}$, a beam diameter of $1.5 \mathrm{~mm}$ at a width of $1 / \mathrm{e}^{2}$ and output power of $5.1 \mathrm{~W}$. As displayed in Figure 1, the laser beam was focused by an F-Theta Scan Lens with a focal length of $160 \mathrm{~mm}$ over the surface of the Ti foil in a batch operation mode while continuously stirred by a magnetic stirrer. The laser beam was moved at a velocity of $2 \mathrm{~m} / \mathrm{s}$ over the foil's surface, employing a dual-axis scanning galvanometer mirror positioning system (intelliSCAN 14, SCANLAB).

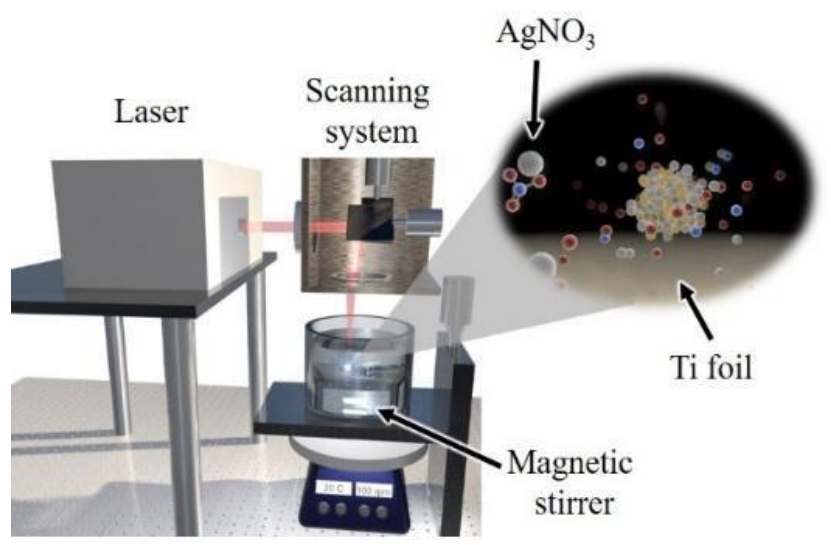

Figure 1 Laser ablation in an aqueous solution of $\mathrm{AgNO}_{3}$ in a batch operation mode

The samples coming from the ablation in the different solutions are labeled as Ti NPs for $0 \mathrm{mM}$, and 0.001 , $0.01,0.1,1$, and $10 \mathrm{mM}$ for the corresponding concentration's solutions. 
After the synthesis, the elemental composition of individual particles was assessed by single-particle inductivecoupled plasma mass spectroscopy (splCP-MS) using a mass spectrometer with inductively coupled plasma (ICP-MS; NexION 3000D, Perkin Elmer). Their crystallographic structure was identified by X-ray diffraction in powder (XRD) using a diffractometer (Miniflex 600, Rigaku) with a wavelength of $1.540 \AA$ operating at a scan speed of $1^{\circ} \cdot \mathrm{min}-1$ in a $2 \theta$ angle range of $30-90^{\circ}$. The ultraviolet-visible (UV-Vis) spectroscopy analysis was performed by acquiring the sample's absorption spectra using a spectrophotometer (DR 3900, Hach-Lange), in a wavelength range from 320 to $1000 \mathrm{~nm}$ with a wavelength resolution of $1 \mathrm{~nm}$. Furthermore, the morphology and size distribution of selected samples were determined using a transmission electron microscope (TEM; JEM-2100Plus, JEOL), which was operated at an acceleration voltage of $200 \mathrm{kV}$.

\section{RESULTS AND DISCUSSION}

\section{1. $\quad$ spICP-MS}

The elemental composition displayed in Table 1, which was assessed in single particles by spICP-MS demonstrates that each element's weight percentage in the synthesized particles is relatively proportional to the $\mathrm{AgNO}_{3}$ solution's concentration, except for the case of $0.01 \mathrm{mM}$, which seems to be comparable to the one observed for $0.1 \mathrm{mM}$. The solution's concentration that approaches the most to a 50 to $50 \mathrm{wt} \%$ elemental composition is $1 \mathrm{mM}$, which also establishes a border of the order of magnitude in the molar solution at which it is possible to control the percentage of $\mathrm{Ag}$ to $\mathrm{Ti}$ in the nanoalloys, thus leading to the design of truly tailored nanoalloys.

Table 1 Average concentration and the corresponding weight percentage of $\mathrm{Ag}$ and $\mathrm{Ti}$ found in the samples by spICP-MS (the weight percentage is provided without considering the contribution of oxygen due to its measurement's inaccuracy while employing spICP-MS).

\begin{tabular}{|c|c|c|c|c|}
\hline Sample & $\begin{array}{c}\text { Ag average concentration } \\
(\mathbf{m g} / \mathbf{L})\end{array}$ & $\begin{array}{c}\text { Ti average concentration } \\
(\mathbf{m g} / \mathbf{L})\end{array}$ & $\begin{array}{c}\mathbf{A g} \\
(\mathbf{w t} \%)\end{array}$ & $\begin{array}{c}\mathbf{T i} \\
(\mathbf{w t} \%)\end{array}$ \\
\hline Ti NPs & 0.0 & 35.35 & 0 & 100 \\
\hline $0.001 \mathrm{mM}$ & 0.14 & 35.20 & 0.40 & 99.60 \\
\hline $0.01 \mathrm{mM}$ & 1.70 & 25.00 & 6.37 & 93.63 \\
\hline $0.1 \mathrm{mM}$ & 2.11 & 31.87 & 6.21 & 93.79 \\
\hline $1 \mathrm{mM}$ & 14.95 & 32.94 & 31.22 & 68.78 \\
\hline $10 \mathrm{mM}$ & 331.62 & 41.14 & 88.96 & 11.04 \\
\hline
\end{tabular}

\subsection{XRD}

Figure 2, which shows the comparison of the XRD patterns found in the different samples, exhibits in accordance with the results from spICP-MS, that the crystalline phases found in the samples with a high concentration of Ti, i.e., Ti NPs, $0.001 \mathrm{mM}, 0.01 \mathrm{mM}$, and $0.1 \mathrm{mM}$, only belong to titanium oxides. On the contrary, the samples with a larger concentration of $\mathrm{Ag}$ also display the $\mathrm{Ag}$ crystalline phase.

For the samples Ti NPs, $0.001 \mathrm{mM}, 0.01 \mathrm{mM}$, and $0.1 \mathrm{mM}$, it is possible to identify the crystalline families (111) at $43.46^{\circ},(200)$ at $50.64^{\circ}$, and $(220)$ at $74.44^{\circ}$, which correspond to the cubic crystalline phase $\mathrm{TiO}_{1.04}$ with a space group $\mathrm{Fm}-3 \mathrm{~m}$, and cell lattice parameters $\mathrm{a}=\mathrm{b}=\mathrm{c}=4.1940 \AA$ (JCPDS database: PDF-00-043-1296). Also, the family (116) at $63.41^{\circ}$ of the rhombohedral crystal phase $\mathrm{Ti}_{2} \mathrm{O}_{3}$ with the space group $\mathrm{R}-3 \mathrm{c}$, and cell lattice parameters $\mathrm{a}=5.139 \AA$, and $\mathrm{c}=13.659 \AA$ (JCPDS database: PDF-10-63).

The sample $1 \mathrm{mM}$, exhibits the presence of the crystalline phase $\mathrm{TiO}_{1.04}$, and the families (111) at $44.8^{\circ},(200)$ at $51.8^{\circ}$, and (220) at $77^{\circ}$, which belong to the crystalline cubic phase of $\mathrm{Ag}$ with a space group $\mathrm{Fm}$ and cell 
lattice parameters: $a=b=c=4.0862 \AA$ (JCPDS database: 00-004-0783). Finally, the sample $10 \mathrm{mM}$ only exhibits the Ag crystalline phase. Unlike the samples synthesized in lower $\mathrm{AgNO}_{3}$ solution concentrations, the peaks belonging to the titanium oxide phases seem to be overshadowed by the once of $\mathrm{Ag}$, which agrees with previous studies where the increment of $\mathrm{Ag}$ wt\% results in higher visibility of the Ag crystalline phase [11].

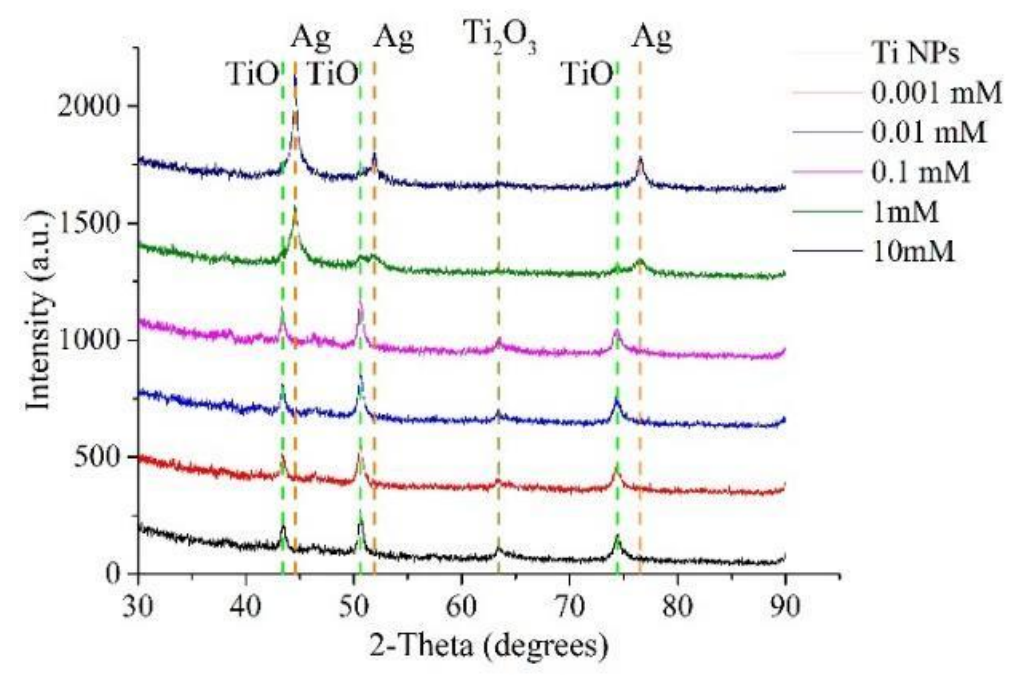

Figure 2 XRD spectra of the synthesized samples. The green vertical dashed lines indicate the TiO crystalline phase peaks, the orange lines the $\mathrm{Ag}$, and dark yellow the $\mathrm{Ti}_{2} \mathrm{O}_{3}$ phases

\subsection{UV-Vis}

The absorbance spectra (Figure 3) demonstrates that the alloying with Ag heavily increments the synthesized particles' absorption in the wavelength region 350-600 nm, providing in this way coexisting absorbance edges centered at 400 and $663 \mathrm{~nm}$ in average, which may potentially enable the transfer of photo-induced charge carriers between the $\mathrm{Ti}$ oxide and $\mathrm{Ag}$. Without the presence of $\mathrm{AgNO}_{3}$, the sample labeled as Ti NPs displays a spectrum found in $\mathrm{Ti}$ NPs with coexisting crystalline phases of various oxides like $\mathrm{TiO}, \mathrm{Ti}_{2} \mathrm{O}_{3}$, and $\mathrm{TiO}_{2}$ that displays an absorbance edge at $400 \mathrm{~nm}$ (typical result when a Ti foil undergoes ablation in water) [12]. Within the addition of $\mathrm{AgNO}_{3}$, the samples start to display an absorption peak that belongs to the SPR activity of $\mathrm{Ag}$ NPs, and with this, the possibility to generate photo-induced charge carriers from a broader range of the electromagnetic spectrum. In the sample labeled $0.001 \mathrm{mM}$, the absorbance maximum is centered at $533 \mathrm{~nm}$ with an edge at $717 \mathrm{~nm}$, which, according to the Mie theory, corresponds to spherical Ag NPs with sizes around $117 \mathrm{~nm}$, or core-shell nanostructure with an $\mathrm{Ag}$ core of $62 \mathrm{~nm}$ and a $\mathrm{TiO}_{2}$ shell of $7 \mathrm{~nm}$ [13]. According to the spICP-MS results, a scenario where particles are mainly composed of Ag seems unlikely; therefore, it is more reasonable to deduce that the use of such minimal $\mathrm{AgNO}_{3}$ solution's concentration does not lead to an adequate elemental mixing and may preferably lead to the separate synthesis of $\mathrm{TiO}_{\mathrm{x}} \mathrm{NPs}$ and $\mathrm{Ag} \mathrm{NPs}$. Moreover, the larger intensity of the titanium oxide crystalline phases and the lack of Ag phase in the XRD measurements support the incorrect mixing hypothesis. In the case of $0.01 \mathrm{mM}$, the Ag NPs SPR peak's presence at $430 \mathrm{~nm}$ and edge at $751 \mathrm{~nm}$ seems to get heavily reduced, which agrees with the behavior that Ag-based alloys should display for the elemental composition found by spICP-MS [14]. In the case of $0.1 \mathrm{mM}$, the plasmon peak is centered at $421 \mathrm{~nm}$ and have the edge at $627 \mathrm{~nm}$, which according to the Mie theory, corresponds either to Ag Nps with sizes around $50 \mathrm{~nm}$, or NPs with a $\mathrm{TiO}_{2}$ core of $6 \mathrm{~nm}$ and Ag shell of $21 \mathrm{~nm}$ [13]. Similar to the case of $0.001 \mathrm{mM}$, according to the spICP-MS results, a scenario where the elements did not mix correctly seems more plausible. In the $1 \mathrm{mM}$ and $10 \mathrm{mM}$ cases, the SPR peaks are located at 433 and $426 \mathrm{~nm}$, and the edges at 630 and $620 \mathrm{~nm}$, respectively. According to the Mie theory, the location and intensity of the SPR peaks correspond to Ag NPs with sizes around $60 \mathrm{~nm}$ or NPs with a $\mathrm{TiO}_{2}$ core of $9 \mathrm{~nm}$ and $\mathrm{Ag}$ 
shell of $26 \mathrm{~nm}$ for $1 \mathrm{mM}$, and Ag NPs with sizes around $55 \mathrm{~nm}$ or NPs with a $\mathrm{TiO}_{2}$ core of $4 \mathrm{~nm}$ and Ag shell of $25 \mathrm{~nm}$ for $10 \mathrm{mM}$. Given the spICP-MS and XRD results, the presence of both elements in individual particles seems more plausible. Therefore, similar to the outcome drawn from the elemental composition analysis, 1 $\mathrm{mM}$ seems to be the solution's concentration threshold at which it is possible to get a high control over the elemental composition and optical properties in the synthesized alloys.

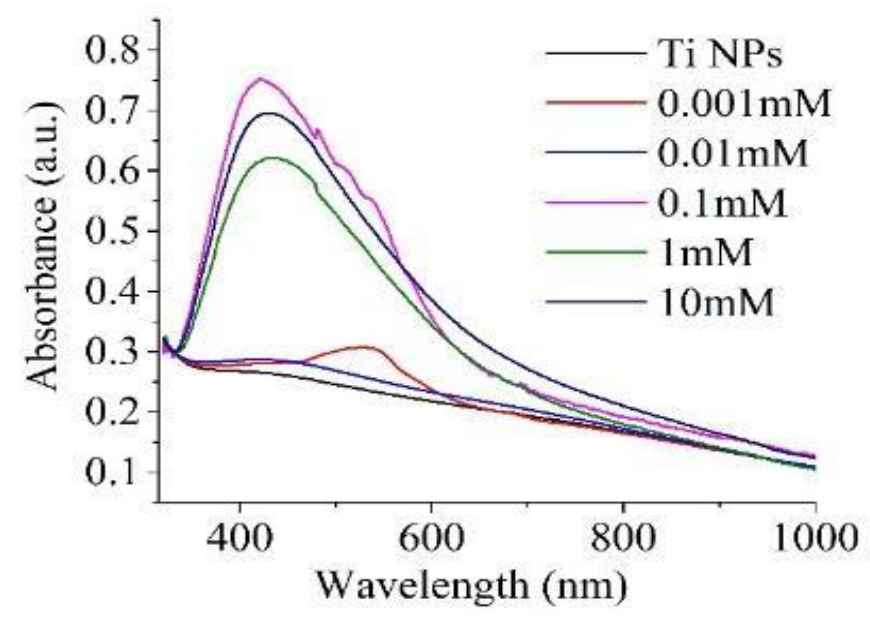

Figure 3 Absorbance spectra of the synthesized samples

\subsection{TEM}

Given the previous results, the samples labeled as Ti NPs and $1 \mathrm{mM}$ were selected for their morphological analysis by means of TEM. Figure 4, which displays a micrograph picture of both samples accompanied by their corresponding size histogram, exhibits two essential aspects that significantly relate to the previous results.
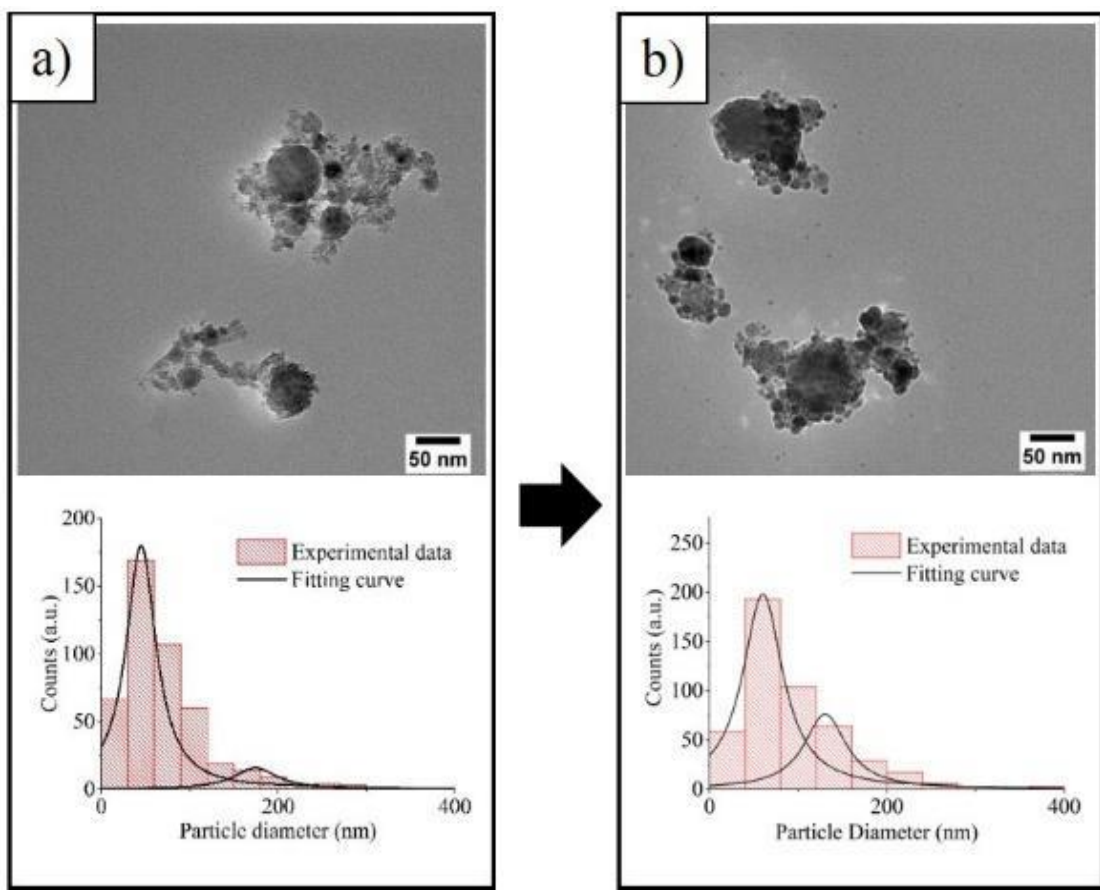

Figure 4 TEM micrograph (top side) and histogram of the size distribution (bottom side) of a) the samples synthesized in pure water and b) in a $1 \mathrm{mM}$ aqueous solution of $\mathrm{AgNO}_{3}$ 
On the one hand, both materials display a bimodal size distribution, which is typical for NPs synthesized by laser ablation in liquid mediums [15]. For Ti NPs, the average size is centered at (45 \pm 20.7$) \mathrm{nm}$, and (174 \pm $30.2) \mathrm{nm}$, while for $1 \mathrm{mM}$ at $(60 \pm 27.6) \mathrm{nm}$, and $(130 \pm 29.1) \mathrm{nm}$. On the other hand, the morphology of the titanium oxide NPs seems to be spine-like for the smaller elements and spherical for the bigger once, while it is spherical for $1 \mathrm{mM}$ whether they are small or big particles. Similar to the case of monovalent salts usage for the quenching in the size of the laser-synthesized NPs, the usage of $1 \mathrm{mM} \mathrm{AgNO}_{3}$ solution's concentration seems to interact intimately with the incoming titanium oxide NPs leading to their reshape in a spherical form [16].

\section{CONCLUSION}

The present study sheds light on the capabilities of the synthesis of NPs based on the ablation of solid targets in liquid media to expand the optical properties of materials such as titanium oxides. The analysis of samples coming from the ablation of a ti foil immersed in various concentrations of $\mathrm{AgNO}_{3}$ aqueous solutions enables determining that $1 \mathrm{~mm}$ is a concentration's threshold where it is possible to control the elemental composition, crystalline structure, and optical absorption of the synthesized spherical alloys. Besides, since the alloying with $\mathrm{Ag}$ provides the $\mathrm{Ti}$ oxide NPs an absorption peak that coincides with the maximum of the solar spectral irradiance, i.e., $350-600 \mathrm{~nm}$, and the coexistence of absorbance edges centered at 400 and $638 \mathrm{~nm}$, it can be concluded that the current methodology increments the capabilities of titanium oxide NPs to harvest and turn into usable energy the radiation coming from widespread sources of energy like the sun.

Overall, the current study will be of great value for modifying titanium oxide NPs to increment their employability in more light-powered applications.

\section{ACKNOWLEDGEMENTS}

\section{The research presented in this article was supported by the Internal Grant of the Technical University of Liberec (SGS-2020-4053)}

\section{REFERENCES}

[1] ZHANG, Q., et al. Nanomaterials for energy conversion and storage. Chemical Society Reviews. 2013, vol. 42, no. 7, pp. 3127-3171.

[2] LUCIANI, G., IMPARATO, C., VITIELLO, G. Photosensitive Hybrid Nanostructured Materials: The Big Challenges for Sunlight Capture. Catalysts. 2020, vol. 10, no. 1, pp. 103.

[3] WANG, Z., et al. H-doped black titania with very high solar absorption and excellent photocatalysis enhanced by localized surface plasmon resonance. Advanced Functional Materials. 2013, vol. 23, no. 43, pp. 5444-5450.

[4] CHEN, X., et al. Increasing solar absorption for photocatalysis with black hydrogenated titanium dioxide nanocrystals. Science. 2011, vol. 331, no. 6018, pp. 746-750.

[5] MEYMIAN, M.R.Z., ABDOLVAHAB, R.H., MEHR, A.K. Fractal characteristics of $\mathrm{TiO}_{2}-\mathrm{Ag}$ nanocomposite films deposited by a grid-assisted co-sputtering method. Applied Surface Science. 2019, vol. 480, pp. 593-600.

[6] DAL'TOÉ, A.T., et al. Lanthanum doped titania decorated with silver plasmonic nanoparticles with enhanced photocatalytic activity under UV-visible light. Applied Surface Science. 2018, vol. 441, pp. 1057-1071.

[7] PRABHU, B., VALANARASU, A. Stability analysis of $\mathrm{TiO}_{2}-\mathrm{Ag}$ nanocomposite particles dispersed paraffin wax as energy storage material for solar thermal systems. Renewable Energy. 2020, vol. 152, pp. 358-367.

[8] ZHANG, D., GÖKCE, B., BARCIKOWSKI, S. Laser synthesis and processing of colloids: fundamentals and applications. Chemical Reviews. 2017, vol. 117, no. 5, pp. 3990-4103.

[9] TORRES-MENDIETA, R., et al. Laser-assisted synthesis of Fe-Cu oxide nanocrystals. Applied Surface Science. 2019, vol. 469, pp. 1007-1015.

[10] MATSUMOTO A., et al. Transfer of the species dissolved in a liquid into laser ablation plasma: an approach using emission spectroscopy. The Journal of Physical Chemistry C. 2015, vol. 119, no 47, pp. 26506-26511. 
[11] GUPTA, K., et al. Photocatalytic antibacterial performance of $\mathrm{TiO}_{2}$ and $\mathrm{Ag}$-doped $\mathrm{TiO}_{2}$ against S. aureus. P. aeruginosa and E. coli. Beilstein Journal of Nanotechnology. 2013, vol. 4, no. 1, pp. 345-351.

[12] HUANG, CH.-N., et al. Nonstoichiometric titanium oxides via pulsed laser ablation in water. Nanoscale Research Letters. 2010, vol. 5, no. 6, pp. 972.

[13] OLDENBURG, S.J. Light Scattering from Gold Nanoshells. Diss. Rice University, 2000.

[14] KULADEEP, R., et al. Laser-assisted synthesis of Au-Ag alloy nanoparticles with tunable surface plasmon resonance frequency. Optical Materials Express. 2012, vol. 2, no. 2, pp. 161-172.

[15] REHBOCK, CH, et al. Current state of laser synthesis of metal and alloy nanoparticles as ligand-free reference materials for nano-toxicological assays. Beilstein Journal of Nanotechnology. 2014, vol. 5, no. 1, pp. 1523-1541.

[16] LETZEL, A., et al. Time and mechanism of nanoparticle functionalization by macromolecular ligands during pulsed laser ablation in liquids. Langmuir. 2019, vol. 35, no. 8, pp. 3038-3047. 